\title{
Entschließung der Informationsfreiheitsbeauftragten der Länder vom 24. April 2017
}

\section{Open Data: Gesetzentwurf der Bundesregierung greift zu kurz!}

Die Informationsfreiheitsbeauftragten der Länder fordern den Deutschen Bundestag auf, statt des von der Bundesregierung vorgelegten Entwurfs eines Open-Data-Gesetzes (Erstes Gesetz zur Änderung des E-Government-Gesetzes) das Informationsfreiheitsgesetz des Bundes zu einem umfassenden Transparenzgesetz zu entwickeln. Bereits im Dezember letzten Jahres hat die Konferenz der Informationsfreiheitsbeauftragten in Deutschland ihre Bedenken angesichts des geplanten Open-Data-Gesetzes in einer Entschließung zum Ausdruck gebracht. Das mittlerweile fortgeschrittene Gesetzgebungsverfahren bietet Anlass, noch einmal ausdrücklich auf folgende Bedenken hinzuweisen:

Der Deutsche Bundestag hat sich am 31. März 2017 in erster Lesung mit dem Entwurf der Bundesregierung für ein Erstes Gesetz zur Änderung des E-Government-Gesetzes (BT-Drucksache 18/11614) befasst. Bund und Länder hatten am 14. Oktober 2016 vereinbart, Open Data zu stärken. Dabei verpflichteten sich die Länder, Open-Data-Gesetze nach dem Beispiel der Bundesregelung zu erlassen. Die Ergebnisse im aktuellen Gesetzgebungsverfahren auf Bundesebene werden daher erhebliche Auswirkungen auf die Landesgesetzgebung haben.

\section{Neben Rohdaten auch Dokumente aktiv veröffentlichen}

Der Entwurf richtet sich ausschließlich auf die Bereitstellung von Rohdaten. Informationen, die aus sich heraus verständlich sind, zum Beispiel Verträge, Gutachten, Stellungnahmen und ähnliche Dokumente, sind davon nicht umfasst. Für das von der Bundesregierung angestrebte Ziel der Stärkung zivilgesellschaftlicher Teilhabe ist dies aber ein entscheidender Gehalt des Gesetzes.

\section{Transparenzregelungen gehören in Transparenzgesetze}

Die Informationsfreiheitsbeauftragten der Länder sind der Ansicht, dass das Informationsfreiheitsgesetz des Bundes der richtige Standort für eine Open-Data-Regelung wäre. Die Aufnahme von Open-Data-Regelungen in das E-Government-Gesetz des Bundes fördert zwar den Open-Data-Gedanken. Dabei darf jedoch nicht übersehen werden, dass die Behörden des Bundes nach wie vor verpflichtet bleiben, amtliche Informationen nach Maßgabe des Informationsfreiheitsgesetzes des Bundes zur Verfügung zu stellen. Eine zusätzliche Einzelregelung für offene Daten passt nicht in das bislang informationstechnisch orientierte E-Government-Gesetz. Statt die Regelung dort einzufügen, sollten die vorgesehenen Regelungen im Informationsfreiheitsgesetz verankert werden. Dieses würde so zu einem modernen Transparenzgesetz, das erforderlichenfalls als weiteres Vorbild für die Landesgesetzgebung dienen könnte. Jede weitere Zersplitterung der ohnehin bereits unübersichtlichen Regelungen zum Informationszugang sollte vermieden werden.

\section{Keine zusätzlichen Ausnahmen}

Der Gesetzentwurf verweist zwar auf die Ausnahmetatbestände des Informationsfreiheitsgesetzes, enthält aber noch weitere Ausnahmen. Beispielsweise sollen nur Daten veröffentlicht werden, die außerhalb der Behörde liegende Verhältnisse betreffen. Das mit dem Gesetzentwurf verfolgte Ziel nach „mehr Teilhabe interessierter Bürgerinnen und Bürger und eine intensivere Zusammenarbeit der Behörde mit diesen" dürfte damit nicht erreicht werden. Es erscheint insgesamt inkonsequent, Open Data durch Ausnahmen zu beschränken, die über die Regelung des Informationsfreiheitsgesetzes hinausgehen. Hiervon ist abzusehen. Die Weiterentwicklung der Informationsfreiheit kann nur im Abbau von Ausnahmen bestehen, nicht in deren Ausweitung.

\section{Individueller Anspruch auf Veröffentlichung}

Der Regierungsentwurf gewährt keinen individuellen Anspruch auf die Veröffentlichung von Daten. Ein solcher Anspruch, der von jedermann einklagbar wäre, ist als effektives Korrektiv zu einer reinen Selbstverpflichtung der öffentlichen Stellen jedoch unverzichtbar.

Für die Länder, die amtliche Informationen auf der Grundlage von Informationsfreiheitsgesetzen bereits in Informationsregistern zur Verfügung stellen, wie auch für die anderen Länder kann das geplante Open-Data-Gesetz in dieser Form keine Vorbildfunktion entfalten. Die Weiterentwicklung des Informationsfreiheitsgesetzes des Bundes zu einem Transparenzgesetz mit den dazugehörigen Open-Data-Regelungen könnte dagegen eine entsprechende Signalwirkung für die Länder haben.

Die Informationsfreiheitsbeauftragten der Länder fordern den Bundestag eindringlich auf, den eingeschlagenen Sonderweg zu überdenken. 